\title{
Impactos psicológicos da pandemia de COVID-19 em profissionais da saúde
}

\author{
Psychological impacts of the COVID-19 pandemic on healthcare professionals \\ Impactos psicológicos de la pandemia COVID-19 em los profesionales de la
}

\author{
Adna Gorette Ferreira Andrade \\ ORCID: https://orcid.org/0000-0003-3847-2890 \\ Faculdade Independente do Nordeste, Brasil \\ E-mail: adnagorette@fainor.com.br \\ Alaiane Paola Almeida Oliveira \\ ORCID: https://orcid.org/0000-0001-5675-8193 \\ Faculdade Independente do Nordeste, Brasil \\ E-mail: alaianepaola@hotmail.com \\ Joice Kelaine Silva Amaral \\ ORCID: https://orcid.org/0000-0003-2324-1061 \\ Faculdade Independente do Nordeste, Brasil \\ E-mail: joicemaral10@gmail.com \\ Umbelina Joane Silva Amaral \\ ORCID: https://orcid.org/0000-0001-8082-2183 \\ Faculdade Independente do Nordeste, Brasil \\ E-mail: belamara153@gmail.com
}

\begin{abstract}
Resumo
Objetivo: Verificar os impactos psicológicos da COVID-19 em profissionais de saúde. Metodologia: Trata-se de uma pesquisa de caráter exploratório, descritivo, com abordagem quantitativa. $\mathrm{O}$ estudo foi realizado em uma instituição privada de ensino superior, localizada na cidade de Vitória da Conquista, Sudoeste da Bahia. Os instrumentos utilizados para coleta de dados foram um questionário sociodemográfico desenvolvido pelos pesquisadores de estudo, questionário DASS-21 (Depression Anxiety Stress) desenvolvida por Lovibond e Lovibond (1995), e, por fim, foi aplicado o questionário de Îndice da Qualidade do Sono de Pittsburgh (PSQI) desenvolvido por Buysse. Resultados: Participaram desta pesquisa 12 profissionais da área da saúde, entre os dados coletados dos participantes, no que tange o nível de Ansiedade, Depressão e Estresse 83,3\% dos entrevistados apresentaram o índice dentro da normalidade, o PSQI, a partir dos escores encontrados na amostra, 58,3\% dos entrevistados apresentaram a qualidade do sono ruim. Conclusão: conclui-se que os níveis de ansiedade, depressão e estresse entre os profissionais de saúde foi considerado predominantemente normal e a frequência da qualidade de sono ruim neste estudo foi alta.
\end{abstract}

Palavras-chave: COVID-19; Angústia psicológica; Pandemia; Profissionais de saúde.

\begin{abstract}
Objective: To verify the psychological impacts of COVID-19 on health professionals. Methodology: This is an exploratory, descriptive research with a quantitative approach. The study was carried out in a private higher education institution, located in the city of Vitoria da Conquista, Southwest of Bahia. The instruments used for data collection were a sociodemographic questionnaire developed by the study researchers, DASS-21 (Depression Anxiety Stress) questionnaire developed by Lovibond and Lovibond (1995), and finally the Pittsburgh Sleep Quality Index questionnaire was applied. (PSQI) developed by Buysse. Results: Twelve health professionals participated in this research, among the data collected from the participants, regarding the level of Anxiety, Depression and Stress, 83.3\% of respondents had the index within normality, the PSQI, based on the scores found in the sample, 58.3\% of respondents had poor sleep quality. Conclusion: it is concluded that the levels of anxiety, depression and stress among health professionals were considered predominantly normal and the frequency of poor sleep quality in this study was high.
\end{abstract}

Keywords: COVID-19; Psychological distress; Pandemic; Health professionals.

\section{Resumen}

Objetivo: Verificar los impactos psicológicos del COVID-19 en los profesionales de la salud. Metodología: Se trata de una investigación exploratoria, descriptiva con enfoque cuantitativo. El estudio se realizó en una institución privada de educación superior, ubicada en la ciudad de Vitoria da Conquista, al suroeste de Bahía. Los instrumentos utilizados para la recolección de datos fueron un cuestionario sociodemográfico desarrollado por los investigadores del estudio, el cuestionario DASS-21 (Depression Anxiety Stress) desarrollado por Lovibond y Lovibond (1995), y finalmente se aplicó el cuestionario Pittsburgh Sleep Quality Index (PSQI) desarrollado por Buysse. Resultados: Doce profesionales 
de la salud participaron en esta investigación, entre los datos recolectados de los participantes, respecto al nivel de Ansiedad, Depresión y Estrés, el $83.3 \%$ de los encuestados presentó el índice dentro de la normalidad, el PSQI, en base a los puntajes encontrados en la muestra, El 58,3\% de los encuestados tenía mala calidad de sueño. Conclusión: se concluye que los niveles de ansiedad, depresión y estrés entre los profesionales de la salud se consideraron predominantemente normales y la frecuencia de mala calidad del sueño en este estudio fue alta.

Palabras clave: COVID-19; Distrés psicológico; Pandemia; Profesionales de la salud.

\section{Introdução}

A propagação de doenças pelo mundo é uma problemática existente há séculos, e hoje com os avanços advindos da globalização, dentre eles a diminuição das fronteiras entres os países, tem se tornado um dos grandes contribuintes para o surgimento de pandemias no mundo (Oliveira, 2020).

Segundo a Organização Mundial de Saúde (OMS, 2020), a pandemia é definida como a disseminação de uma determinada doença que se espalha rapidamente em diversas regiões, continental ou mundial, com transmissão sustentada de pessoa para pessoa. Hoje o mundo enfrenta um grande problema de saúde pública do século que é a COVID-19, doença causada pelo novo coronavírus (SARS-CoV-2), que se originou em Wuhan província de Hubei na China (Brito; Mendes; \& Santos, 2018; Schueler, 2020).

O diretor-geral da Organização Mundial da Saúde (OMS), Tedros Adhanom Ghebreyesus, anunciou em 11 de março de 2020 que a COVID-19 foi caracterizada como pandemia, e segundo a OMS atualmente foram confirmados no mundo 258.830.438 casos de Coronavírus 5.174.646 mortes. É importante salientar, que essa doença trouxe consigo diversas consequências, dentre elas os impactos psicológicos nos profissionais que desenvolvem suas atividades laborais frente ao cenário da pandemia de COVID-19 (OPAS, 2020; Santos et al., 2020).

Esses impactos estão correlacionados com sintomas de estresse, ansiedade, depressão, insônia e sofrimento psicológico em geral. Essas alterações psicológicas estão associadas a vários fatores, dentre eles a pouca informação sobre o surto em seu início, pouco apoio social disponível, carga horaria de trabalho excessivo, distanciamento de familiares e amigos, equipamentos de proteção inadequados e alta taxa de infecção entre as equipes (Martins \& Ferreira, 2020).

É válido ressaltar, que devido a esses fatores que os profissionais estão submetidos, é de extrema importância o cuidado e proteção com a saúde mental destes, pois é significativo o risco de alterações psicológicas diante de tal situação (Teixeira et al., 2020). Dessa forma, justifica-se a realização desse estudo, cujo objetivo é avaliar os impactos psicológicos nos profissionais de saúde que continuam desenvolvendo suas atividades laboras frente ao cenário da pandemia de COVID-19.

\section{Metodologia}

O presente estudo fundamentou-se em uma pesquisa de caráter exploratório, descritivo, com abordagem quantitativa. Esta abordagem tem como foco principal o coletivo, ou seja, a predominância de uma característica em grupo, promovendo assim o fortalecimento da estatística (Mussi, 2019; Knechtel, 2014). Segundo Franco, Dantas (2017), a pesquisa exploratória se integra na primeira etapa de um estudo, tendo como objetivo aperfeiçoar hipóteses, validar instrumentos, bem como buscar uma melhor familiarização com o campo de estudo.

O estudo foi realizado em uma instituição privada de ensino superior, localizada na cidade de Vitória da Conquista, Sudoeste da Bahia. A escolha do local foi por conveniência, bem como pela oferta de cursos da área da saúde que a faculdade proporciona. A população de estudo foi representada por profissionais da saúde, que também atuam como supervisores de estágio em ambientes de baixa a alta complexidade (fisioterapeutas, enfermeiros e farmacêuticos). Participaram deste estudo, profissionais de ambos os gêneros da área da saúde, que atuam em ambientes hospitalares, ambulatoriais e/ou farmácias, e que 
tenham mais de um ano de prática clínica. Foram excluídos da pesquisa aqueles profissionais que se encontram de férias, folgas, licenças, ou ainda, cumprindo aviso prévio.

A pesquisa esteve de acordo com o que preconiza as normas éticas e legais exigidas pela resolução $n^{\circ} 466 / 2012$ (Conselho Nacional de Saúde). O projeto foi submetido ao Comitê de Ética em Pesquisa da Faculdade Independente do Nordeste (CEP/FAINOR), sendo que a coleta só foi iniciada após aprovação e autorização do CEP-FAINOR, sob parecer de $\mathrm{n}^{\mathrm{o}}$ 4.958.453.

Os instrumentos utilizados para coleta de dados foram, um questionário sociodemográfico desenvolvido pelos pesquisadores de estudo, com intenção de identificar os seguintes dados: gênero, raça, renda, idade e estado civil. Também foi investigado informações laborais dos profissionais participantes do estudo, como: formação, tempo e área de atuação, carga horária de trabalho, se atuam também em outros ambientes que prestam serviço à saúde e carga horária nestes serviços.

Posteriormente, foi aplicado um questionário DASS-21 (Depression Anxiety Stress) desenvolvida por Lovibond e Lovibond (1995), validada em português por Escalas de Ansiedade Depressão e Stress (EADS 21). Segundo Martins et al. (2019) a EADS se divide em três escalas, sendo elas: Depressão, Ansiedade e Stress, sendo que cada uma delas são constituídas por 7 itens, tendo um total 21 itens, o resultado de cada escala é determinado pela soma dos resultados dos sete itens. A escala apresenta três notas, sendo elas: uma por sub-escala, onde o mínimo é " 0 " e o máximo " 21 ". As maiores notas em cada escala vão corresponder a estados afetivos mais negativos.

Por fim, foi aplicado o questionário de Índice da Qualidade do Sono de Pittsburgh (PSQI) desenvolvido por Buysse et al. (1989), sendo um instrumento de confiabilidade, validade, simples e bem aceito pelos pacientes. Esse questionário é composto por 19 itens, onde são agrupados em sete componentes, sendo pontuados em uma escala de 0 a 3 . Ao final, os escores dos sete componentes são somados, onde a pontuação varia de 0 a 21. Pontuações de 0-4 indicam boa qualidade do sono, de 5-10 indicam qualidade ruim e acima de 10 indicam distúrbio do sono

Os instrumentos foram transcritos para plataforma do Google Forms pelos pesquisadores para serem preenchidos/assinalados pelos participantes de forma não presencial, ou seja, aquele contato por meio ou ambiente virtual, não envolvendo a presença física das pesquisadoras, seguindo as orientações das Resoluções do Conselho Nacional de Saúde $\mathrm{n}^{\circ}$ 466 de 2012 e a de $n^{\circ} 510$ de 2016. Os dados coletados foram transcritos em dupla entrada em banco de dados eletrônico, no programa Excel versão 2019 e, transportado para o programa estatístico, onde procedeu a análise de cada variável.

\section{Resultados}

Participaram desta pesquisa 12 profissionais da área da saúde que também atuam como supervisores dos estágios obrigatórios, sendo eles; $50 \%$ fisioterapeutas, $25 \%$ farmacêuticos e $25 \%$ enfermeiros, a maioria pertence ao sexo feminino (66,6\%), 58,3\% se autodeclararam como pardas e apresentam o estado civil como casadas (75\%). Quando questionado sobre a renda mensal, 91,6\% afirmaram receber mais de 3 salários-mínimos e exercer uma carga horária semanal menor ou igual a 60 horas semanais, declarando também que trabalham em outros vínculos além do cargo de supervisor de estágio, conforme dados demonstrados da Tabela 1. 
Tabela 1 - Perfil socioeconômico dos participantes da pesquisa, Vitória da Conquista, 2021.

\begin{tabular}{cc}
\hline Variáveis & $\mathbf{\%}$ \\
Sexo & $\mathbf{6 6 , 6 \%}$ \\
Feminino & $\mathbf{3 3 , 3 \%}$ \\
Masculino & \\
Raça & $\mathbf{5 8 , 3 \%}$ \\
Parda & $\mathbf{3 3 , 3 \%}$ \\
Branca & $\mathbf{8 , 3 \%}$ \\
Estado Civil & $\mathbf{2 5 \%}$ \\
Solteiro(a) & $\mathbf{7 5 \%}$ \\
Casado (a) & $\mathbf{9 1 , 6 \%}$ \\
Renda mensal & $\mathbf{8 , 3 \%}$ \\
Mais de 3 salários-mínimos & $\mathbf{5 0 \%}$ \\
1 a 3 salários-mínimos & $\mathbf{2 5 \%}$ \\
Formação & $\mathbf{2 5 \%}$ \\
Fisioterapeuta & \\
Enfermeiro(a) & $\mathbf{4 1 , 6 \%}$ \\
Farmacêutico (a) & $\mathbf{4 1 , 6 \%}$ \\
= 60 horas & $\mathbf{1 6 , 6 \%}$ \\
< 60 horas & \\
$>$ 60 horas & $\mathbf{6 6 , 6 \%}$ \\
Outros vínculos & $\mathbf{3 3 , 3 \%}$ \\
Sim &
\end{tabular}

Fonte: Dados da Pesquisa.

Em seguida foi aplicado o segundo instrumento avaliativo denominado como questionário DASS-21 (Depression, Anxiety Stress), desenvolvida por Lovibond e Lovibond (1995), validada em português por Escalas de Ansiedade Depressão e Stress (EADS 21). Entre os dados coletados dos participantes, no que tange o nível de Ansiedade, Depressão e Estresse 83,3\% dos entrevistados apresentaram o índice dentro da normalidade, como mostra a Tabela 2.

Tabela 2 - Classificação sobre o nível de Depressão, Ansiedade e Estresse (DASS 21), Vitória da Conquista, 2021.

\begin{tabular}{ccc}
\hline & Classificação & $\%$ \\
\hline \multirow{2}{*}{ DEPRESSÃO } & Normal & 83,3 \\
& Leve & - \\
& Moderado & 8,3 \\
& Severo & 8,3 \\
\multirow{2}{*}{ ANSIEDADE } & Normal & 83,3 \\
& Leve & - \\
& Moderado & 8,3 \\
& Severo & 8,3 \\
& & 83,3 \\
& Normal & 8,3 \\
& Leve & - \\
& Moderado & 8,3 \\
\hline
\end{tabular}

Fonte: Dados da Pesquisa.

Por fim, na análise do Índice de Qualidade do Sono destes profissionais, através do PSQI, a partir dos escores encontrados na amostra, 58,3\% dos entrevistados apresentaram a qualidade do sono ruim (Tabela 3). 
Tabela 3 - Índice da Qualidade do Sono de Pittsburgh (PSQI), Vitória da Conquista, 2021.

\begin{tabular}{cc}
\hline Variável & $\%$ \\
\hline Boa qualidade do sono & 25 \\
Qualidade do sono ruim & 58,3 \\
Distúrbio do sono & 16,6 \\
\hline
\end{tabular}

Fonte: Dados da Pesquisa.

\section{Discussão}

No presente estudo, houve uma predominância do sexo feminino com 66,6\%, o que pode ser justificado pelo fato de que a área da saúde tem uma predominância deste sexo. Segundo Moreira (1999), as relações de trabalho masculino sempre se relacionavam com a força física e à abstração, ao contrário do sexo feminino onde estavam ligadas à sensibilidade e à observação, podendo justificar a grande inserção das mulheres como profissionais de saúde, por ser uma área que exige dos profissionais muita atenção e grande sensibilidade. De acordo com Hernandes e Vieira (2020), a feminização da força de trabalho na saúde vem abrangendo as principais categorias em relação a ações da saúde da população, sendo que o trabalho feminino corresponde a 78,9\% do trabalho na área da saúde.

Foi observador em relação a renda mensal que 91,6\% dos profissionais recebem mais de três salários-mínimos mensalmente, corroborando com esse resultado, Elias e Navarro (2006) aborda que é possível observar que muitos profissionais fazem uma dupla jornada de trabalho devido à má remuneração salarial que a classe de profissionais de saúde vem recebendo, fazendo com que esses trabalhadores se esforcem mais para alcançarem um melhor salário.

De acordo com Gomes e Coqueiro (2017), a carga horária semanal de trabalho dos profissionais de saúde tendem a variar dependendo da instituição, alguns lugares são trinta, trinta e seis, quarenta e quarenta e quatro horas semanais, sabendo que a jornada de trinta e seis horas é a mais comum. Sendo que o limite máximo de jornada semanal destes profissionais é de 60 horas. Diante disso foi observado neste estudo que apenas 16,6\% dos profissionais ultrapassam o limite máximo de 60 horas, os demais fazem $60(41,6 \%)$ ou menos que 60 horas semanais $(41,6 \%)$, sendo assim os resultados evidenciam que a maioria dos participantes se encaixam dentro da normalidade de carga horária semanal.

Segundo Appel, Carvalho e Santos (2021), diante dos longos turnos de trabalho, a experiência em conviver com inúmeras mortes por conta da COVID - 19, bem como, uma grande demanda de pacientes em processo de tratamento lutando para se manterem vivos, podem agravar ainda o grau estresse na desses profissionais que atuam em ambientes hospitalares. E essas vulnerabilidades causadas no ambiente de trabalho vem desencadeando preocupação aos profissionais da saúde, que se tornam susceptíveis a níveis elevados de ansiedade e depressão, estes, considerados como os males do século XXI.

Diante disso, o presente estudo objetivou investigar os níveis de ansiedade, depressão e estresse entre profissionais que atuam na linha de frente da COVID-19. Sendo assim, foi aplicado a escala DASS-21, onde não foi possível verificar nos entrevistados, a partir dos instrumentos da pesquisa, níveis elevados de ansiedade, depressão e estresse, baseado nos fatores investigados. Para todos os eixos avaliavam a ansiedade, depressão e estresse a pontuação final dos participantes apresentaram um escore de normalidade, representado por $83,3 \%$ da amostra. Contrariamente a esse estudo, a pesquisa de Chew et al (2020), realizada com profissionais em um hospital de Cingapura e Índia, evidenciou escores moderados a muito graves no que diz respeito a ansiedade, depressão e estresse. Em contrapartida, o estudo de Abreu et al (2021), realizado com profissionais durante a pandemia, evidenciou que o sintoma de estresse, diante aos outros quesitos avaliados apresentou uma maior prevalência.

Segundo Serrão et al. (2007), o sono assume um elemento fundamental no que tange a recuperação física e psicológica do ser humano. É de suma importância para obter uma boa qualidade de vida, onde permite uma restauração física, 
além de proteger do desgaste natural das horas acordadas. Diante disso, foi utilizado como ferramenta para mensurar e classificar a qualidade do sono da amostra - O Índice de qualidade de sono de Pittsburgh (PSQI).

Os resultados evidenciados nesta pesquisa chamam atenção no que diz respeito ao escore da qualidade de sono ruim dos participantes, obtendo um percentual de 58,3\%. Seguindo esse raciocínio, Serra (2014), aborda que as alterações no sono podem causar consequências no que diz respeito ao nível do desequilíbrio emocional, podendo assim desencadear transtornos depressivos, de ansiedade e estresse. Freitas e Costa em um estudo realizado em 2020, aborda resultados semelhantes com o esse estudo, demonstrando valores relevantes e preocupantes com a qualidade de sono, abordando que grande parte da amostra estudada entre os profissionais possuem uma qualidade de sono ruim. Santos et al (2021) em seu estudo, realizado em um Hospital Público de Teresina-PI, durante o período de pandemia, trouxe resultados semelhantes, abordando que os participantes apresentaram uma má qualidade de sono também.

Desta forma, medidas podem ser adotadas, sendo que a proteção de saúde mental dos profissionais é de grande importância para evitar alterações psicológicas, como também melhorar a atuação deles no ambiente de trabalho. É importante a disponibilidade de EPI'S adequados, carga horaria adequada e acompanhamento psicológico principalmente para aqueles mais suscetíveis (Schmidt et al; 2020; Saidel et al; 2020).

\section{Conclusão}

De acordo com os resultados obtidos, conclui-se que os níveis de ansiedade, depressão e estresse entre os profissionais de saúde foi considerado predominantemente normal segundo a Depression, Anxiety, Stress Scales (DASS-21), porém diante a longos turnos de trabalho, inúmeras mortes, podem transformar sintomas leves em graves consequências para a saúde. Sendo assim, independente do resultado, faz-se necessário um olhar cuidadoso para com esses profissionais.

A frequência da qualidade de sono ruim neste estudo foi alta, isso pode trazer uma baixa capacidade para desempenhar as atividades do dia a dia e do trabalho. Diante disto é imprescindível a inclusão de sessões de educação para a saúde sobre o sono. A adoção desta prática pode contribuir para um melhor aproveitamento do período disponível, assim como um melhor desempenho no trabalho.

Faz -se necessário a elaboração de estudos mais amplos para mensurar os impactos psicológicos causados nos profissionais da saúde na pandemia, visto que este tema ainda é pouco estudado. Devido o estudo ter apresentado algumas fragilidades como: uma amostra pequena de participantes, bem como a realização do questionário de forma remota o que pode ter contribuído para que alguns participantes não participassem devido alguma intercorrência.

\section{Referências}

Abreu, L. et al (2021). Rastreio de sintomas de depressão, ansiedade e estresse em fisioterapeutas durante a pandemia do COVID-19. Ver Movimenta, 14, 107119. < https://www.revista.ueg.br/index.php/movimenta/article/view/11196/8329>

Appel, A.., Carvalho, A., \& Santos, R. (2021). Prevalência e fatores associados à ansiedade, depressão e estresse numa equipe de enfermagem COVID-19. Rev. Gaúcha Enferm. 42. https://doi.org/10.1590/1983-1447.2021.20200403

Brito, G, Mendes, A, \& Santos, P. (2018). objeto de trabalho na estratégia saúde da família. Interface: Communication, Health, Education, 22(64). https://doi.org/10.1590/1807-57622016.0672

Buysse, D. et al. (1989). O índice de qualidade do sono de Pittsburgh: um novo instrumento para a pratica e pesquisa psiquiátrica. Psychiatry Res. 28 (2): 193213. < https://pubmed.ncbi.nlm.nih.gov/2748771/>

Chew, N. W. S. et al (2020). Um estudo multinacional e multicêntrico sobre os resultados psicológicos e sintomas físicos associados entre profissionais de saúde durante COVID-19 surto. Brain, Behavior, and Immunity 88 559-565. https://doi.org/10.1016/j.bbi.2020.04.049.

Elias, M.., \& Navarro, V. (2006). A relação entre o trabalho, a saúde e as condições de vida: negatividade e positividade do trabalho das profissionais de enfermagem de um hospital escola. Rev. Latino-Am. Enfermagem 14 (4). https://doi.org/10.1590/S0104-11692006000400008 
Franco, M., \& Dantas, O. (2017). Pesquisa exploratória: aplicando instrumentos de geração de dados- observação, questionário e entrevista. (16). . http://docplayer.com.br/63965456-Pesquisa-exploratoria-aplicando-instrumentos-de-geracao-de-dados-observacao-questionario-e-entrevista.html>

Freitas, A, Costa, C. M. (2020). Qualidade do sono, risco de ansiedade e depressão e comportamento alimentar de médicos e enfermeiros em uma unidade publica localizada na cidade de Maracanaú-CE. < http://repositorio.unifametro.edu.br/bitstream/123456789/712/1/AMANDA\%20SAMPAIO\%20FREITAS\% 20e\%20CAR LOS\%20MANUEL\%20FONTENELE\%20PAULINO\%20DA\%20COSTA_TCC.pdf>

Gomes, R., \& Coqueiro, J. (2017). Qualidade de Vida Relacionada à Carga de Trabalho dos Profissionais de Saúde com enfoque nos problemas desencadeados. Id on Line Rev. Psic. 10(33). https://idonline.emnuvens.com.br/id/article/view/617>

Hernandes, E., \& Viera, L. (2020). A guerra tem rosto de mulher: trabalhadoras da saúde no enfrentamento a Covid-19. < http://anesp.org.br/todas-asnoticias/2020/4/16/a-guerra-tem-rosto-de-mulher-trabalhadoras-da-sade-no-enfrentamento-covid-19>

Knechtel, M. (2014). Metodologia da pesquisa em educação: uma abordagem teórica- pratica dialogada. Curitiba. Intersaberes. 193. < https://doi.org/10.5212/PraxEduc.v.11i2.0013>

Martins, B. et al. (2019). Escala de Depressão, Ansiedade e Estresse: propriedades psicométricas e prevalência das afetividadesJ. bras. psiquiatr. 68(1). https://doi.org/10.1590/0047-2085000000222

Martins, H, \& Ferreira, B. (2020). Evidencias cientificas sobre os impactos psicológicos de epidemias/pandemias em profissionais de saúde. Psic., Saúde \& Doenças 21(3). <https://doi.org/10.15309/20psd210309>.

Moreira, M. (1999). Imagens no espelho de Vênus: mulher, enfermagem e modernidade. Rev Latino-Am Enfermagem. 7(1), 55-65. https://www.scielo.br/j/rlae/a/mxHybkx9Ss6bD44DkMmyNVm/?format=pdf\&lang=pt>

Mussi, R. et al. (2019). Pesquisa Quantitativa e/ou Qualitativa: distanciamentos, aproximações e possibilidades. Revista Sustinere, 7(2), 414-430. https://www.e-publicacoes.uerj.br/index.php/sustinere/article/view/41193/32038>

Oliveira, M. (2020). Globalização, saúde e meio ambiente: a pandemia da COVID-19. Edital simplificado de fluxo contínuo. https://www.uern.br/controledepaginas/PPGEO-Inser\%C3\%A7\%C3\%A3o\%20Social/arquivos/5542globalizaa\%E2\%80\%A1a\%C6\%92o_ saa\% C5\%A1de_ e_meio_am biente_a_pandemia_da_covid_19.pdf>

OPAS - Organização Pan-Americana da Saúde.(2020) Folha informativa COVID-19 - Escritório da OPAS e da OMS no Brasil. Brasília, https://www.paho.org/pt/covid19>

Santos, J. S. et al (2021). A qualidade de sono de fisioterapeutas de um hospital publico durante a pandemia de COVID-19. Rev. Pesqui. Fisioter. 11, 510-517. http://dx.doi.org/10.17267/2238-2704rpf.v11i3.3911

Santos, W et al. (2020). O impacto da pandemia da COVID-19 na saúde mental dos profissionais de saúde: revisão integrativa. Research, Society and Development, 8, (9), e190985470. < https://doi.org/10.31011/reaid-2020-v.93-n.0-art.758>

Saidel, M. et al. (2020). Intervenções em saúde mental para profissionais de saúde frente a pandemia de Coronavírus. Rev enferm UERJ, (28), e49923. Retrived from < https://pesquisa.bvsalud.org/portal/resource/pt/biblio-1097213>

Schmidt, B. et al (2020). Saude mental e intervenções psicológicas diante da pandemia do novo coronavirus (COVID-19). Estud. psicol. 37. < https://doi.org/10.1590/1982-0275202037e200063>

Schueler, P. (2020). O que é uma pandemia. Fiocruz, https://www.bio.fiocruz.br/index.php/br/noticias/1763-o-que-e-uma-pandemia>

Serra, F. (2014). Efeitos da crise económica na saúde mental: Portugal na União Europeia (2004-2012). Editora FEUC. < https://estudogeral.uc.pt/handle/10316/28070>

Serrão, F., Klein, J, \& Gonçalves, A. (2007), Qualidade do sono e depressão: que relações sintomáticas em crianças de idade escolar. Psico-USF, 12 (2), https://doi.org/10.1590/S1413-82712007000200014

Teixeira, C. F. et al. (2020). A saúde dos profissionais de saúde no enfrentamento da pandemia de Covid-19. Ciencia \& saude coletiva, $25(9)$, 3465-3474. https://www.cienciaesaudecoletiva.com.br/artigos/a-saude-dos-profissionais-de-saude-no-enfrentamento-da-pandemia-de-covid19/17634?id=17634 